Canad. Math. Bull. Vol. 19 (2), 1976

\title{
THE UNIFORM CONTINUITY OF FUNCTIONS IN SOBOLEV SPACES
}

\author{
BY \\ R. A. $\mathrm{ADAMS}^{(1)}$
}

\begin{abstract}
Functions in $W^{m, p}(\Omega) \cap W_{0}^{1, q}(\Omega), m p>\operatorname{dim} \Omega, q \geq 1$, may have to be uniformly continuous on $\Omega$ even if $\Omega$ is not a Lipschitz domain.
\end{abstract}

1. Introduction. Let $\Omega$ be a domain (an open set) in $n$-dimensional Euclidean space $\mathbf{R}^{n}$. We denote the boundary of $\Omega$ by $\partial \Omega$. The Sobolev space $W^{m, p}(\Omega)$ consists of (equivalence classes of) functions $u$ in $L^{p}(\Omega)$ whose distributional derivatives $D^{\alpha} u$ also belong to $L^{p}(\Omega)$ whenever $|\alpha| \leq m$. ( $m$ is a positive integer; $p$ is real, $p \geq 1 ; \alpha=\left(\alpha_{1}, \ldots, \alpha_{n}\right)$ is an $n$-tuple of nonnegative integers; $\left.|\alpha|=\alpha_{1}+\cdots+\alpha_{n} ; D^{\alpha}=\left(\partial / \partial x_{1}\right)^{\alpha_{1}} \cdots\left(\partial / \partial x_{n}\right)^{\alpha_{n}}.\right) W^{m, p}(\Omega)$ is a Banach space with respect to the norm

$$
\|u\|_{m, p, \Omega}=\left\{\sum_{|\alpha| \leq m} \int_{\Omega}\left|D^{\alpha} u(x)\right|^{p} d x\right\}^{1 / p} .
$$

$W_{0}^{m, p}(\Omega)$ is the closure in $W^{m, p}(\Omega)$ of the space $C_{0}^{\infty}(\Omega)$ of infinitely differentiable functions having compact support in $\Omega$.

We denote by $C(\bar{\Omega})$ the space of functions $u$ bounded and uniformly continuous on $\Omega$ and having, therefore, unique continuous extensions to the closure $\bar{\Omega}$ of $\Omega$, and by $C_{B}(\Omega)$ the space of functions bounded and continuous on $\Omega$. Both are Banach spaces with respect to the norm $\sup _{x \in \Omega}|u(x)|$.

The domain $\Omega$ has the cone property if there exists an open, finite, right spherical cone $C$ such that each point $x \in \Omega$ is the vertex of a finite cone $C_{x}$ contained in $\Omega$ and congruent to $C . \Omega$ is a Lipschitz domain if each point $x \in \partial \Omega$ has a neighbourhood $U_{x}$ such that, for some rectangular coordinate system $\xi$ in $U_{x}, U_{x} \cap \Omega$ is specified by an inequality of the form $\xi_{n}<f\left\{\xi_{1}, \ldots, \xi_{n-1}\right)$ where $f$ is a Lipschitz continuous function.

Many imbedding results for $W^{m, p}(\Omega)$ can be obtained under the fairly mild requirement that $\Omega$ should have the cone property. For instance, for such $\Omega$, $W^{m, p}(\Omega)$ is imbedded in $C_{B}(\Omega)$ provided $m p>n$. (This is a part of the "Sobolev

Received by the editors August 12, 1974.

(1) Research partially supported by the National Research Council of Canada under Operating Grant number A-3973.

AMS 1970 Subject Classification-Primary 46E35. 
Imbedding Theorem"- -see e.g. [1], theorem 5.4.) Certain imbeddings, however, require more regularity of $\Omega$. One cannot in general expect to imbed $W^{m, p}(\Omega)$ into $C(\bar{\Omega})$ if $\Omega$ has only the cone property. Two obvious counterexamples are the split squares:

$$
\begin{aligned}
& \Omega_{1}=\left\{x=\left(x_{1}, x_{2}\right) \in \mathbf{R}^{2}:-1<x_{1}<1,0<\left|x_{2}\right|<1\right\} \\
& \Omega_{2}=\Omega_{1} \cup\left\{x \in \mathbf{R}^{2}:-1<x_{1}<0, x_{2}=0\right\} .
\end{aligned}
$$

Both $\Omega_{1}$ and $\Omega_{2}$ have the cone property and $\Omega_{2}$ is connected. However the reader may readily construct a function $u$ belonging to $W^{m, p}(\Omega)\left(\Omega=\Omega_{1}\right.$ or $\left.\Omega_{2}\right)$ for every $m, p$, but which satisfies $\lim _{x_{2} \rightarrow 0-} u(x) \neq \lim _{x_{2} \rightarrow 0+} u(x)$ for $x_{1}>0$, and hence cannot be uniformly continuous on $\Omega$.

If $\Omega$ is a bounded Lipschitz domain then the Sobolev imbedding theorem assures us that $W^{m, p}(\Omega)$ is imbedded in $C(\bar{\Omega})$ provided $m p>n$. We examine circumstances under which the Lipschitz property can be weakened. It is clear, at least for bounded $\Omega$, that elements of $C_{B}(\Omega)$ which also happen to tend to zero on $\partial \Omega$ belong to $C(\bar{\Omega})$. Since for any $q$ the elements of $W_{0}^{1, q}(\Omega)$ may be regarded as vanishing "in a generalized sense" on $\partial \Omega$ (see Lemma 2 below) one is led to the conjecture:

$$
\mathrm{W}^{\mathrm{m}, \mathrm{p}}(\Omega) \cap \mathrm{W}_{0}^{1, \mathrm{q}}(\Omega) \subset \mathrm{C}(\bar{\Omega}) .
$$

There is good reason to suspect that this conjecture is true for arbitrary domains $\Omega$ (see section 5 below) but this writer has been unable to discover a general proof. We can prove it for arbitrary domains with the cone property using a well-known theorem of E. Gagliardo [4] on the decomposition of such domains into unions of Lipschitz domains.

THEOREM 1. Let $\Omega$ be a domain in $\mathbf{R}^{n}$ having the cone property. If $m p>n$ and $q \geq 1$ then $W^{m, p}(\Omega) \cap W_{0}^{1, q}(\Omega) \subset C(\bar{\Omega})$. More generally, for any nonnegative integer $j, W^{m+j, p}(\Omega) \cap W_{0}^{1+j, q}(\Omega) \subset C^{j}(\bar{\Omega})$.

Here, of course, $C^{j}(\bar{\Omega})$ denotes the space of functions $u$ for which $D^{\alpha} u \in$ $C(\bar{\Omega})(|\alpha| \leq j)$, normed by $\max _{|\alpha| \leq j} \sup _{x \in \Omega}\left|D^{\alpha} u(x)\right|$. Theorem 1 need only be proved for $j=0$ as it then follows for general $j$ be application of the special case to derivatives $D^{\alpha} u,|\alpha| \leq j$. We give a proof in sections 3 and 4 below. At this point we can make several remarks.

(i) Theorem 1 is only of interest when $q \leq n$. If $q>n$ it is a trivial consequence of the Sobolev imbedding theorem that $W_{0}^{1+j, q}(\Omega)$ is imbedded in $C^{j}(\bar{\Omega})$ for arbitrary domains $\Omega$ (since zero extension outside $\Omega$ imbeds $W_{0}^{k, q}(\Omega)$ into $W^{k, q}\left(\mathbf{R}^{n}\right)$ ). Several useful characterizations of $W_{0}^{k, q}(\Omega)$ for $q>n$ are known (see Burenkov $[2,3])$ but these are of no avail in the context of our problem.

(ii) It is not difficult to find examples of domains $\Omega$ not having the cone property for which, at least for some of the appropriate values of $m, p$ and $q$ 
the conclusion of Theorem 1 holds. (See section 5.) It is for this reason that we conjecture that Theorem 1 may hold for arbitrary domains, but a different sort of proof will be necessary to show this.

(iii) The (generalized) vanishing of functions is not really required on the whole of the boundary of $\Omega$ for Theorem 1 to hold. One might consider replacing $W_{0}^{1, q}(\Omega)$ by the larger space $W_{0}^{1, q}\left(\Omega^{*}\right)$, the closure in $W^{1, q}(\Omega)$ of the space of infinitely differentiable functions of compact support in $\mathbf{R}^{n}$ which vanish near $\partial \Omega \sim \partial \bar{\Omega}$. It is clear, for instance, that such is the case for the two examples $\Omega_{1}$ and $\Omega_{2}$ given above, where for each we have $\partial \Omega \sim \partial \bar{\Omega}=$ $\left\{x \in \partial \Omega: x_{2}=0\right.$ and $\left.-1<x_{1}<1\right\}$.

(iv) Weak solutions of null Dirichlet problems for elliptic partial differential equations on $\Omega$ are known a priori to belong to spaces of the form $W_{0}^{k, q}(\Omega)$ (usually with $q=2$ ). Theorem 1 thus enables us to obtain "up to the boundary" regularity of solutions in $W^{m, p}(\Omega)$ for suitably large $m p$ even if $\Omega$ has only the cone property.

2. A preliminary lemma. Before proving Theorem 1 we prepare the following lemma. It is well-known, at least for smoothly bounded domains, and asserts that continuous functions in $W_{0}^{1,1}(\Omega)$ do in fact vanish on sufficiently well-behaved parts of $\partial \Omega$.

LEMMA 2. Let $\Omega$ be a domain in $\mathbf{R}^{n}$ and $G$ a bounded Lipschitz domain contained in $\Omega$. Let $u \in W_{0}^{1,1}(\Omega) \cap C(\bar{G})$ and let $x \in \partial G$. If there exists a neighbourhood $N$ of $x$ such that $N \cap \partial G \subset \partial \Omega$ then $u(x)=0$.

Proof. Suppose $u(x) \neq 0$. We may select the neighbourhood $N$ small enough that $|u(x)| \geq \delta>0$ for $x \in N \cap \bar{G}$. By virtue of the Lipschitz property of $G$ we may, again contracting $N$ if necessary, find a nonzero vector $y$ such that for all $z \in N \cap \partial G$ and all $s, 0<s<1$, we have $z+s y \in G$. Without loss of generality $y=k(0,0, \ldots, 0,1)$. Let $V=\{z+s y: z \in N \cap \partial G, 0<s<1\}$. Writing $z=\left(z^{\prime}, z_{n}\right)$ where $z^{\prime}=\left(z_{1}, \ldots, z_{n-1}\right)$, setting $P=\left\{z^{\prime}:\left(z^{\prime}, z_{n}\right) \in N \cap \partial G\right.$ for some $\left.z_{n}\right\}$, and denoting by $z_{n}^{*}$ the unique number which, for given $z^{\prime} \in P$, satisfies $\left(z^{\prime}, z_{n}^{*}\right) \in$ $N \cap \partial G$, we have

$$
V=\left\{z=\left(z^{\prime}, z_{n}\right): z^{\prime} \in P, z_{n}^{*}<z_{n}<z_{n}^{*}+k\right\} .
$$

Let $\phi \in C_{0}^{\infty}(\Omega)$ and set $v=u-\phi$. Then $\left|v\left(z^{\prime}, z_{n}^{*}\right)\right|=\left|u\left(z^{\prime}, z_{n}^{*}\right)\right| \geq \delta$ for all $z^{\prime} \in P$. If $z=\left(z^{\prime}, z_{n}\right) \in V$ then

$$
v\left(z^{\prime}, z_{n}\right)=v\left(z^{\prime}, z_{n}^{*}\right)+\int_{z_{n}^{*}}^{z_{n}} \frac{\partial}{\partial s} v\left(z^{\prime}, s\right) d s
$$

whence

$$
\delta \leq\left|v\left(z^{\prime}, z_{n}\right)\right|+\int_{z_{n}^{*}}^{z_{n}^{*+k}}\left|\frac{\partial}{\partial s} v\left(z^{\prime}, s\right)\right| d s
$$


Integrating $z$ over $V$ we obtain

$$
\begin{aligned}
(\operatorname{vol} V) \delta & \leq \int_{V}|v(z)| d z+k \int_{V}\left|\frac{\partial}{\partial z_{n}} v(z)\right| d z \\
& \leq(1+k)\|v\|_{1,1, V} \leq(1+k)\|u-\phi\|_{1,1, \Omega} .
\end{aligned}
$$

Since $u \in W_{0}^{1,1}(\Omega)$ the right side of the last inequality can be made arbitrarily small for suitable choice of $\phi$ and we have a contradiction. Thus $u(x)=0$.

We remark that the above lemma extends with no change in proof to more general domains $G$ than bounded Lipschitz ones. For instance, it is sufficient that $G$ have the segment property. (See [1], section 4.2.)

In view of Lemma 2 the proof of Theorem 1 for domains (like $\Omega_{1}$ above) which are unions of finitely many pairwise disjoint bounded Lipschitz domains is trivial. Similar ad hoc techniques will yield the result for somewhat more complicated domains (e.g. $\Omega_{2}$ ) as well, but for the general case we require the following theorem of E. Gagliardo [4]. (See also, [1], theorem 4.8)

THEOREM 3. (Gagliardo) (a) If $\Omega$ is a bounded domain with the cone property then $\Omega$ is a finite union of bounded Lipschitz domains.

(b) Any domain $\Omega$ (bounded or not) having the cone property is a union of finitely many subdomains each of which is a union of parallel translates of some open parallelepiped.

3. Proof of Theorem 1 for bounded domains. For the time being we assume that $\Omega$ is bounded. Thus $W_{0}^{1, q}(\Omega) \subset W_{0}^{1,1}(\Omega)$ and we may also assume that $q=1$.

As noted above, we may write $\Omega=\bigcup_{V \in \mathscr{F}} V$ where $\mathscr{F}$ is a finite family of bounded Lipschitz subdomains of $\Omega$. Given $u \in W^{m, p}(\Omega) \cap W_{0}^{1,1}(\Omega)$ we have $u \in C_{B}(\Omega)$ and $u \in C(\bar{V})$ for every $V \in \mathscr{F}$. We must show that $u \in C(\bar{\Omega})$.

Let $\nu$ be the number of elements of $\mathscr{F}$ and let $B$ be an open ball in $\mathbf{R}^{n}$. Let $V, W \in \mathscr{F}$ be such that $V \cap B \neq \varnothing$ and $W \cap B \neq \varnothing$. By a $(B, \mathscr{F})$-chain linking $V$ and $W$ we mean any (finite) sequence $\left\{U_{1}, \ldots, U_{k}\right\} \subset \mathscr{F},(k \leq \nu)$, such that $U_{1}=V, \quad U_{k}=W$ and $\bar{U}_{j} \cap U_{j+1} \cap \Omega \cap B \neq \varnothing, 1 \leq j \leq k-1$. Given $V \in \mathscr{F}$ let $\mathscr{A}(V)$ denote the collection of elements $W \in \mathscr{F}$ linked to $V$ by a $(B, \mathscr{F})$-chain. Evidently $W \in \mathscr{A}(V)$ if and only if $V \in \mathscr{A}(W)$.

Let $\varepsilon>0$ be given. For each $V \in \mathscr{F}$ there exists $\delta_{V}>0$ such that if $x, y \in \bar{V}$ and $|x-y|<\delta_{V}$ then $|u(x)-u(y)|<\varepsilon / \nu$. (In this context we regard $u$ as its unique continuous extension to $\bar{V}$.) Let $\delta=\min _{V \in \mathscr{F}} \delta_{V}$ Let $x, y \in \Omega$ satisfy $|x-y|<\delta$. We show that $|u(x)-u(y)|<\varepsilon$ and hence complete the proof.

Let $B$ be an open ball in $\mathbf{R}^{n}$ having diameter $\delta$ and containing $x$ and $y$. There exist elements $V, W \in \mathscr{F}$ such that $x \in V$ and $y \in W$.

Case I. $W \in \mathscr{A}(V)$. In this case there exists a $(B, \mathscr{F})$-chain $\left\{U_{1}, \ldots, U_{k}\right\}$ linking $V=U_{1}$ and $W=U_{k}$. Select points $z_{1}, \ldots, z_{k-1}$ with $z_{j} \in$ 
$\bar{U}_{j} \cap \bar{U}_{j+1} \cap \Omega \cap B$. Evidently

$$
|u(x)-u(y)| \leq\left|u(x)-u\left(z_{1}\right)+\sum_{j=1}^{k-2}\right| u\left(z_{j}\right)-u\left(z_{j+1}\right)|+| u\left(z_{k-1}\right)-u(y) \mid<\varepsilon .
$$

Case II. $W \notin \mathscr{A}(V)$. Then $\mathscr{A}(W) \cap \mathscr{A}(V)=\varnothing$. Let $\lambda, \mu$ be the numbers of elements in $\mathscr{A}(\mathrm{V})$ and $\mathscr{A}(W)$ respectively, so that $\lambda+\mu \leq \nu$. Let $S=$ $\bigcup_{U \in \mathscr{A}(V)} U, T=\bigcup_{U \in \mathscr{A}(W)} U$. We show that there exist points $z \in B \cap \bar{S}, \rho \in$ $B \cap \bar{T}$ such that $u(z)=u(\rho)=0$. Granted this, for the moment, we have $z \in B \cap \bar{U}$ for some $U \in \mathscr{A}(V)$. Hence there exists a $(B, \mathscr{F})$-chain $\left\{U_{1}, \ldots, U_{k}\right\}$ $(k \leq \lambda)$ linking $U_{1}=V$ and $U_{k}=U$. Selecting $z_{1}, \ldots, z_{k-1}$ as in case $I$ we conclude that

$$
|u(x)|=|u(x)-u(z)|<\lambda \varepsilon / \nu .
$$

A similar argument yields $|u(y)|<\mu \varepsilon / \nu$ whence $|u(x)-u(y)|<\varepsilon$ as required It is sufficient, therefore, to show the existence of $z \in B \cap \bar{S}$ with $u(z)=0$.

Let $G \in \mathscr{A}(V)$ and let $\tilde{G}=\bigcup_{U \in \mathscr{A}(V), U \neq G} U$. Thus $S=G \cup \tilde{G}$. Suppose that $t \in B \cap(\partial G \sim \tilde{G})$. Then $t \in \bar{G} \subset \bar{\Omega}$ so that either $t \in \partial \Omega$ or $t \in \Omega$. Since $G$ is open $t \notin G$; thus $t \notin S$. If $t \in \Omega$ then $t \in U$ for some $U \in \mathscr{F}$. Thus $t \in \bar{U} \cap \bar{G} \cap \Omega \cap B$ whence $U \in \mathscr{A}(V)$ and $U \subset S$, a contradiction. Thus $t \in \partial \Omega$ and we have proved

$$
B \cap(\partial G \sim \tilde{G}) \subset \partial \Omega \text { for every } G \in \mathscr{A}(V) .
$$

Now $\partial S=\bigcup_{G \in \mathscr{A}(V)}(\partial G \sim \tilde{G})$ so that

$$
B \cap \partial S \subset \partial \Omega \text {. }
$$

Let $k$ be the largest integer such that every point of $B \cap \partial S$ belongs to the boundaries of at least $k$ distinct elements of $\mathscr{A}(V)$. Clearly $1 \leq k \leq \lambda$. Then there exists $z \in \partial S \cap B$ and elements $G_{1}, \ldots, G_{k} \in \mathscr{A}(V)$ such that $z \in$ $\partial G_{1} \cap \cdots \cap \partial G_{k}$ but $z \notin \bar{G}$ for any $G \in \mathscr{A}(V), G \neq G_{1}, \ldots, G_{k}$. Since $B \sim$ $\bigcup_{G \in \mathscr{A}(V), G \neq G_{1}, \ldots, G k} \bar{G}$ is open, there exists a neighbourhood $N$ of $z$ with $N \subset B$ such that $N \cap \partial S=N \cap \partial G_{1} \cap \cdots \cap \partial G_{k}$ and $N \cap S=N \cap\left(G_{1} \cup \cdots \cup G_{k}\right)$. We show that $N \cap \partial S=N \cap \partial G_{1}$.

Suppose that $a \in N \cap \partial G_{1}$ but $a \notin \partial S$. Evidently $a \in S$ (since otherwise $a \in \operatorname{ext} S$ so $a$ would have a neighbourhood contained in $N$, containing a point of $G_{1}$ but disjoint from $S$ ). It follows that $k \geq 2$ and $a \in G_{j}$ for some $j, 2 \leq j \leq k$. We may assume that $N$ has been chosen so small that $N \cap G_{1}$ lies on one side of a Lipschitz graph in $N$. Let $s \in N \sim S$. We may find a continuous path in $N$ going from $s$ to $a$ which meets $\bar{G}_{1}$ for the first time at $a$. The path meets $\bar{S}$ for the first time at a point of $\partial S \cap N \subset \partial G_{1} \cap \cdots \cap \partial G_{k}$ so this point must be $a$. Since $a \notin \partial S$ we have a contradiction. Hence

$$
N \cap \partial G_{1}=N \cap S \subset \partial \Omega .
$$

It follows from Lemma 2 that $u(z)=0$ and the proof of Theorem 3 for bounded domains is complete. 
4. Extension to unbounded domains. By Theorem 3(b) even an unbounded domain $\Omega$ can be written as a union of finitely many subdomains $\Omega_{j}(1 \leq j \leq k)$ each of which is a union of parallel translates of a fixed open parallelepiped $P_{j}$ having one vertex at the origin; say

$$
\Omega_{j}=\bigcup_{x \in A_{j}}\left(x+P_{j}\right), \quad 1 \leq j \leq k .
$$

The dimensions of $P_{j}$ depend only on the cone $C$ determining the cone property for $\Omega$.

Let $\mathbf{R}^{n}=\bigcup_{\beta} Q_{\beta}$ be a tesselation of $\mathbf{R}^{n}$ into closed cubes of edge length $\rho$ and set

$$
A_{j \beta}=A_{j} \cap Q_{\beta} ; \quad \Omega_{j \beta}=\bigcup_{x \in A_{j \beta}}\left(x+P_{j}\right) .
$$

Evidently $\Omega=\bigcup_{j, \beta} \Omega_{j \beta}$ (no longer necessarily a finite union) and for any $\delta>0$ there exists an integer $R=R(n, \rho, \delta, C)$ such that any ball of diameter $\delta$ intersects at most $R$ of the sets $\Omega_{j \beta}$. It is also shown in the proof of Gagliardo's theorem that for $\rho$ sufficiently small (depending only on the dimensions of the parallelepipeds $P_{j}$ and thus on $C$ ) each $\Omega_{j \beta}$ is a bounded Lipschitz domain; in fact $\left(x+P_{j}\right) \cap\left(y+P_{j}\right) \neq 0$ for every $x, y \in A_{j \beta}$.

For given $u \in W^{m, p}(\Omega), m p>n$, and given $\varepsilon>0$ it is shown in the proof of the imbedding theorem (see, for example, [1] lemma 5.17) that there exists $\delta>0$ depending only on $\varepsilon,\|u\|_{m, p, \Omega}$, and the cone $C$, such that if $x, y \in \Omega_{j \beta}$ for some $j, \beta$ and $|x-y|<\delta$ then $|u(x)-u(y)|<\varepsilon$.

With these observations the proof of Theorem 1 for bounded domains extends to arbitrary domains-one uses in place of $\nu$ the number $R=$ $R(n, \rho, 1, C)$; in place of $\mathscr{F}$ the collection $\left\{\Omega_{j \beta}: \Omega_{j \beta} \cap B_{1} \neq \varnothing\right\}$ where $B_{1}$ is a ball of unit diameter containing $B$. (We assume $\delta \leq 1$.) The remaining details are left to the reader.

5. An example. We conclude by showing that Theorem 1 may hold, at least in part, for domains not having the cone property. Specifically, we consider 2-dimensional domains of the following type:

$$
\Omega=\left\{x=\left(x_{1}, x_{2}\right) \in \mathbf{R}^{2}: 0<x_{1}<a, 0<x_{2}<f\left(x_{1}\right)\right\}
$$

where the positive, increasing function $f$ satisfies

$$
\lim _{x_{1} \rightarrow 0+} \frac{f\left(x_{1}\right)}{x_{1}}=0
$$

so that $\Omega$ has a cusp at the origin.

Given $X, 0<X<a$, we set $\Omega_{X}=\{x \in \Omega: x>X\}$. Then $\Omega_{X}$ is a bounded Lipschitz domain, and if we are given $u \in W^{m, p}(\Omega) \cap W_{0}^{1,1}(\Omega)$ where $m p>2$ we 
may conclude at once that for any $X$ we have $u \in C\left(\overline{\Omega_{X}}\right)$ and $u(x)=0$ for $x \in \partial \Omega_{X} \cap \partial \Omega$. In order to conclude that $u \in C(\bar{\Omega})$ it is evidently sufficient to show that $\lim _{x \in \Omega, x \rightarrow 0} u(x)=0$.

First suppose that $p>2$. Let $x=\left(x_{1}, x_{2}\right) \in \Omega$ be given. For $x_{1}$ sufficiently small the open triangle $T$ with vertices at $\left(x_{1}, x_{2}\right),\left(x_{1}, 0\right)$ and $\left(x_{1}+x_{2}, 0\right)$ lies in $\Omega$. Let $(r, \theta)$ denote polar coordinates of an arbitrary point of $\Omega$ with respect to $x$ as pole. The bottom edge of $T$ has equation $r=g(\theta),-\pi / 2 \leq \theta \leq-\pi / 4$, where $0<g(\theta)<\sqrt{ } 2 x_{2}<\sqrt{ } 2 f\left(x_{1}\right)$. Denoting by $v$ the function $u$ expressed in terms of these polar coordinates, and applying Hölder's inequality to the identity

$$
u(x)=v(0, \theta)=-\int_{0}^{g(\theta)} \frac{d}{d t} v(t, \theta) d t
$$

we obtain

$$
\begin{aligned}
|u(x)|^{p} & \leq \int_{0}^{g(\theta)}\left|\frac{d}{d t} v(t, \theta)\right|^{p} t d t \cdot\left\{\int_{0}^{g(\theta)} t^{-1 /(p-1)} d t\right\}^{p-1} \\
& \leq K_{p}\left[f\left(x_{1}\right)\right]^{p-2} \int_{0}^{g(\theta)}\left|\frac{d}{d t} v(t, \theta)\right|^{p} t d t,
\end{aligned}
$$

where $K_{p}$ depends only on $p$. Integration of $\theta$ from $-\pi / 2$ to $-\pi / 4$ leads to the estimate

$$
\begin{aligned}
|u(x)|^{p} & \leq \frac{4 K_{p}}{\pi}\left[f\left(x_{1}\right)\right]^{p-2} \int_{T}|\operatorname{grad} u(y)|^{p} d y \\
& \leq K_{p}^{\prime}\left[f\left(x_{1}\right)\right]^{p-2}\|u\|_{m, p, \Omega}^{p} .
\end{aligned}
$$

Hence $\lim _{x \in \Omega, x \rightarrow 0} u(x)=0$ in this case.

The case $m p>2, p \leq 2$ remains to be considered; we may assume $m=2$. The technique used above cannot be generalized to involve a repeated integral of the second derivative of $v$ since grad $u$ is not known to vanish on the lower edge of $T$. The following ad hoc argument will yield the desired result providing $p>4 / 3$. Let $R$ be a rectangle of breadth $b$ and height $h \leq 1$. A change of variable mapping $R$ onto a rectangle of breadth $b$ and unit height yields the following form of the norm inequality for the imbedding of $W^{1, p}(R)$ into $L^{q}(R), q=2 p /(2-p)(q$ finite if $p=2)$.

$$
\|w\|_{0, q R} \leq K h^{-1 / 2}\|w\|_{1, p, R}
$$

where $K$ may depend on $b$ but is independent of $h$. Note that $q>2$ if $p>1$. For $x_{1}$ sufficiently small the open rectangle $R$ having vertices at $\left(x_{1}, 0\right)$, $\left(x_{1}, f\left(x_{1}\right)\right),\left(x_{1}+(a / 2), f\left(x_{1}\right)\right)$ and $\left(x_{1}+(a / 2), 0\right)$ is contained in $\Omega$ and contains $T$. 
Since $b=a / 2$ and $h=f\left(x_{1}\right)$ for this rectangle we obtain

$$
\begin{aligned}
\|u(x)\|^{q} & \leq K_{q}^{\prime}\left[f\left(x_{1}\right)\right]^{q-2}\|u\|_{1, q, R}^{q} \\
& \leq K_{q}^{\prime} K\left[f\left(x_{1}\right)\right]^{q-2}\left[f\left(x_{1}\right)\right]^{-q / 2}\|u\|_{2, p, R}^{q} \\
& \leq K_{p}^{\prime \prime}\left[f\left(x_{1}\right)\right]^{(q-4) / 2}\|u\|_{2, p, \Omega}^{q} .
\end{aligned}
$$

We may conclude that $u(x) \rightarrow 0$ as $x \rightarrow 0, x \in \Omega$ provided $q>4$, that is, provided $p>4 / 3$.

The method of this example can, of course, be extended to more general cusp domains but it remains uncertain whether the conclusion of Theorem 1 is valid in its entirety for arbitrary domains.

\section{REFERENCES}

1. R. A. Adams, Sobolev Spaces, Academic Press, New York, 1975.

2. V. I. Burenkov, The approximation of functions in Sobolev spaces by functions of compact support on an arbitrary open set. Dokl. Akad. Nauk CCCP, 202 (1972) 259-262. Engl. Transl. Soviet Math. Dokl. 13 (1972) 60-64.

3. V. I. Burenkov, The approximation of functions in the space $W_{p}^{r}(\Omega)$ for arbitrary open sets $\Omega$ by function with compact support. (Russian). Studies in the theory and applications of differentiable functions of several variables, V. Trudy. Mat. Inst. Steklov 131 (1974), 51-63.

4. E. Gagliardo, Proprietà di alcune classi di funzioni in più variabili, Ric. Mat., 7 (1958), $102-137$.

DePartment of MATHEMATics,

The UNIVERsity OF British COLUMBIA,

Vancouver, B.C., Canada V6T 1W5 